\title{
Glucosides of Pinosylvin and its Monomethyl Ether
}

\author{
B E N G'T L I N D B ER G \\ Organisk-kemiska Institutionen, Kungl. Tekniska Högskolan, Stockholm, Sueden
}

$\mathrm{T}$

he heart wood of Scots pine (Pinus silvestris) and most, perhaps all, members of the genus Pinus contain fungicidic stilbene derivatives, pinosylvin and its monomethyl ether ${ }^{1}$. Glucosides of these phenols have not been isolated from natural sources but the rhizome of the rhubarb (Rheum rhaponticum ) contains a similar stilbene derivative in the form of a glucoside (rhapontin) ${ }^{2}$. If glucosides of pinosylvin and its monomethyl ether occur naturally the isolation, undoubtedly, would be facilitated by a knowledge of their physical properties.

The presence of pinosylvin phenols in the heart wood can be demonstrated by illumination with ultraviolet light, when the heart wood shows a more or less brilliant violet fluorescence. The deposition of pinosylvin in the heart wood is the result of the activity of the cambium. It could well be conceived that this activity is displayed not only in a centripetal direction but also in a centrifugal. As a matter of fact the inner part of the bark adjoining the cambium generally exhibits the same fluorescence as the heart wood. The cause of this fluorescence is at present being studied at this institute.

Pinosylvin, and still more its monomethyl ether are very sparingly soluble in water and this is a complicating factor in the measurement of their toxicity to wood destroying fungi. The glucosides would presumably be more readily soluble and since it may be anticipitated that these fungi have glucosidases at their disposal, the mycological investigation of pinosylvin glucosides is a matter of interest.

The syntheses of the glucosides followed conventional lines. The acetylated derivatives were prepared from the hydroxy stilbenes employing the silver oxide-quinoline method and then deacetylated. In the case of the pinosylvin monoglucoside it was necessary to protect one of the hydroxyl groups. This was accomplished by benzoylation. 
It was not possible, however, to obtain the glucosides $\beta$-pinosylvin monomethyl ether glucoside I, $\beta, \beta^{\prime}$-pinosylvin diglucoside II and $\beta$-pinosylvin monoglucoside III in a distinctly crystalline form, but the analytical results, including catalytic hydrogenation, indicated a fair degree of purity.



Docent Rennerfelt, Statens Skogsforskningsinstitut, Stockholm, has been kind enough to test the biological activity of these preparations and reports that neither pinosylvin monomethyl ether glucoside (slightly soluble in water) nor pinosylvin diglucoside (easily soluble in water) possesses any fungicidic activity to wood destroying fungi (Pullularia pullulans and Polyporus annosus).

\section{EXPERIMENTAL}

Pinosylvin and pinosylvin monomethyl ether were isolated by the method given by Erdtman ${ }^{3}$.

Tetracetyl-p-pinosylvin monomethyl ether glucoside

Pinosylvin monomethyl ether $(2.26 \mathrm{~g})$ and acetobromoglucose $(5.36 \mathrm{~g})$ were dissolved in anhydrous quinoline $(6 \mathrm{ml})$ in a small beaker. Active silver oxide $(2.8 \mathrm{~g})$ was added and the mixture was cooled with ice and stirred for 20 minutes. It was then allowed to stand in a desiccator for an hour. Acetic acid $(20+10 \mathrm{ml})$ was then added and the mixture was well stirred, centrifuged and filtered into water $(600 \mathrm{ml})$. The precipitate was filtered, washed with water, dissolved in benzene and dried over calcium chloride. The brown benzene solution was filtered through a column of aluminium oxide (Brockmann). Most of the colour was removed and the solution obtained was now slightly yellow. It was concentrated under reduced pressure and precipitated with petrol ether. An oil was obtained which slowly solidified. Yield $5.2 \mathrm{~g} \mathrm{(93 \% ).} \mathrm{From} \mathrm{hot} \mathrm{methanolic}$ solution it precipitated as a voluminous gel, which could be dried to an amorphous powder of m. p. $89-90^{\circ}$. When a dilute methanolic solution was allowed to evaporate slowly at room temperature, the substance was obtained as colourless needles. It was also obtained in this form when a dilute solution in benzene was precipitated with petrol ether. M. p. $106-107^{\circ}[\alpha]_{\mathrm{D}}^{20}-24^{\circ}$ in chloroform *.

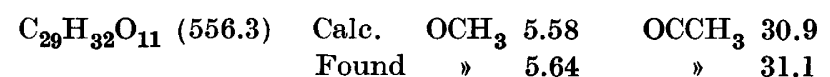

* All melting points uncorrected. All rotations in about $2 \%$ solutions. 


$$
\text { B-Pinosylvin monomethylether glucoside }
$$

Tetraacetyl- $\beta$-pinosylvin monomethyl ether glucoside $(1 \mathrm{~g})$ was dissolved in ethanol $(25 \mathrm{ml}$ ) and a small piece of potassium (about $30 \mathrm{mg}$ ) was added. The solution was boiled up and allowed to stand for two hours. It was then diluted with water $(40 \mathrm{ml})$, acidified with acetic acid and the ethanol distilled off. On cooling a voluminous precipitate was formed, which was filtered and dried, first on porous pot, then over sulphuric acid in a vacuum. Yield $0.59 \mathrm{~g}(84 \%)$. After one further recrystallization from ethanol and two from ethyl acetate it had m. p. 168-169. When dried for several days over phosphorus pentoxide at $70^{\circ}$ in a vacuum it had m. p. $177-178^{\circ}$ and $[a]_{D}^{20}-58^{\circ}$ in acetone-water, $1: 1$. The substance forms a white powder which appears to be amorphous even under the microscope. The m.p. however is sharp and different preparations had the same melting point, so the substance might be microcrystalline.

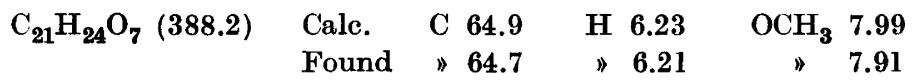

On catalytic hydrogenation in ethanol with palladium on animal charcoal as catalyst 1 mole $\beta$-pinosylvine monomethyl ether glucoside consumed 1.00 mole hydrogen.

A small amount of the glucoside was acetylated with acetic anhydride in pyridine, the solution poured into water and the precipitate recrystallized from benzene-petrol ether. M. p. 106-107. The mixed m. p. with the acetate described above was 106$107^{\circ}$.

\section{Octacetyl- $\beta, \beta^{\prime} \cdot$ pinosylvin diglucoside}

Octaacetyl- $\beta, \beta^{\prime}$-pinosylvin diglucoside was prepared in exactly the same way as tetraacetyl- $\beta$-pinosylvin monomethyl ether glucoside. With pinosylvine (1.06 g), acetobromoglucose $(5.36 \mathrm{~g})$, quinoline $(6 \mathrm{ml})$ and silver oxide $(2.8 \mathrm{~g})$ a sample of slightly discoloured crystals of $\mathrm{m}$. p. $173-174^{\circ}$ was obtained after one recrystallization from ethanol. Yield $2.7 \mathrm{~g}(62 \%)$. After three further recrystallizations from ethanol the material had m. p. $174-175^{\circ}$ and $[a]_{D}^{20}-25^{\circ}$ in chloroform. It forms colourless needles, slightly soluble in ethanol.

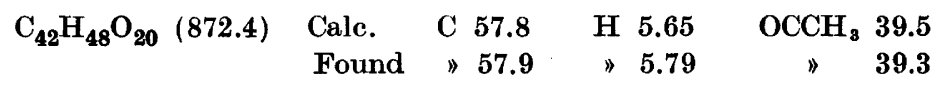

\section{$\beta, \beta$-Pinosylvin digl ucoside}

Octaacetyl- $\beta, \beta^{\prime}$-pinosylvin diglucoside $(2.7 \mathrm{~g})$ was deacetylated with potassium ethoxide in ethanol and the solution titrated with perchloric acid to $\mathrm{pH} \mathbf{7 . 5 5}$ in order to remove the potassium ${ }^{4}$. The potassium perchlorate was filtered off and the ethanol evaporated under reduced pressure. The residue, a slight yellow amorphous powder, could not be recrystallized. Yield $1.65 \mathrm{~g}(100 \%)$. When dried in a vacuum it became somewhat darker, if the temperature was raised to $70^{\circ}$ it became dark brown. It is easily soluble in water and acetic acid, very slightly soluble in ethanol and practically insoluble in anhydrous organic solvents such as ether and benzene. It has no melting point but slowly decomposes at $140^{\circ} .[a]_{\mathrm{D}}^{20}-87^{\circ}$ in acetone-water, $1: 1$. 


$$
\begin{array}{llll}
\mathrm{C}_{26} \mathrm{H}_{32} \mathrm{O}_{12} \text { (536.3) } & \text { Calc. C } 58.2 & \text { H } 6.02 \\
& \text { Found " } 57.9 & \text { " } 6.15
\end{array}
$$

On catalytic hydrogenation 1 mole glucoside consumed 0.97 mole hydrogen

A small amount of the glucoside was acetylated with acetic anhydride in pyridine and the resulting product recrystallized from ethanol. M. p. 174-175 . Mixed m.p. with the octaacetate described above $174-175^{\circ}$.

\section{Pinosylvin monobenzoate}

Pinosylvin (5.8 g) was dissolved in a mixture of absolute ether $(100 \mathrm{ml})$ and absolute pyridine $(10 \mathrm{ml})$. Benzoyl chloride $(3.16 \mathrm{ml})$ in ether $(50 \mathrm{ml})$ was slowly added with vigourous mechanical stirring. The ether was washed with $2 \mathrm{~N}$ hydrochloric acid (200 $\mathrm{ml})$ and with water $(200 \mathrm{ml})$. Ether was added to a final volume of $1000 \mathrm{ml}$ and the solution extracted with $0.3 \%$ sodium hydroxide. From this alkaline solution pinosylvin $(1.6 \mathrm{~g})$ was recovered. The ether was distilled and the residue recrystallized from ethanol. Crude pinosylvin dibenzoate $(2.0 \mathrm{~g})$ separated. The mother liquor was evaporated to dryness. When the residue was recrystallized from xylene, a second crop of crude pinosylvin monobenzoate $(3.2 \mathrm{~g})$, m. p. $110-115^{\circ}$, was obtained. After two recrystallizations from xylene the m. p. was $118-120^{\circ}$. From the mother liquors further amounts of pure monobenzoate could be recovered. Total yield $2.7 \mathrm{~g}, \mathrm{~m} . \mathrm{p} .118-120^{\circ}$.

Pinosylvin monobenzoate $(207.7 \mathrm{mg})$ was acetylated with acetic anhydride in pyridine ( $2 \mathrm{ml}$ ), the amount equivalent to $45.65 \mathrm{ml} 0.0996 \mathrm{~N}$ sodium hydroxide. After the acetylation the mixture was poured into water and titrated. $39.22 \mathrm{ml} 0.0996 \mathrm{~N}$ sodium hydroxide were consumed.

The difference, $6.43 \cdot 0.0996 \mathrm{~m}$. equivalents, has been consumed by the pinosylvin monobenzoate.

$$
\mathrm{C}_{21} \mathrm{H}_{16} \mathrm{O}_{3} \text { (316.1) Found equiv. wt. } 324
$$

Pinosylvin monobenzoate $(100 \mathrm{mg})$ was methylated with diazomethane in ether. The excess of diazomethane was destroyed with acetic acid and the ether evaporated. The yield of pinosylvin monomethyl ether benzoate was quantitative. The substance was recrystallized from acetone-methanol. M. p. 82-84. The substance prepared by Erdtman ${ }^{1}$ by benzoylation of pinosylvin monomethyl ether had m.p. $84.5-86^{\circ}$ and the mixed m. p. of the two preparations was $83-85^{\circ}$.

\section{Pinosylvin monobenzoate acetate}

Pinosylvin monobenzoate was acetylated with acetic anhydride in pyridine (see above). When the mixture was poured into water, colourless crystals appeared. The suspension was extracted with ether, the ether solution shaken with dilute hydrochloric acid, sodium bicarbonate and water, dried over sodium sulphate and finally filtered through a column of aluminium oxide. The ether was distilled and the remaining oil soon solidified, yielding colourless crystals. They were recrystallized twice from ethanol. M. p. $131-132.5^{\circ}$.

$$
\begin{array}{lll}
\mathrm{C}_{23} \mathrm{H}_{18} \mathrm{O}_{4}(358.1) & \text { Calc. C 77.1 } & \text { H } 5.07 \\
& \text { Found " 77.0 } & \text { " } 5.01
\end{array}
$$


Tetraacetyl- $\beta$-pinosylvin monobenzoate glucoside

Pinosylvin monobenzoate $(1.64 \mathrm{~g})$ and acetobromoglucose $(5.36 \mathrm{~g})$ were dissolved in quinoline and silver oxide $(2.8 \mathrm{~g})$ was added. The mixture was stirred for 20 minutes and then allowed to stand in a desiccator for 1 hour. Acetic acid $(20+10 \mathrm{ml})$ was then added and the mixture was well stirred and centrifuged. The liquid phase was filtered into 600 $\mathrm{ml}$ water. The precipitate was filtered, washed with water and dissolved in benzene. The benzene solution was dried over calcium chloride, filtered through a column of aluminium oxide and concentrated in a vacuum. The residue could not be recrystallized but was obtained as a white, amorphous powder by precipitating an ethanolic solution with water. Yield $2.8 \mathrm{~g}(83 \%)$. M. p. $63-65^{\circ}$ (unsharp). $[\alpha]_{\mathrm{D}}^{20}-20^{\circ}$ (chloroform).

$$
\begin{array}{llllcl}
\mathrm{C}_{35} \mathrm{H}_{34} \mathrm{O}_{12} \text { (646.3) } & \text { Cale. C } 65.0 & \text { H } & 5.31 & \text { Acyl groups } 5 \\
& \text { Found " } 64.1 & \text { " } 5.40 & \text { " } & & 5.15
\end{array}
$$

The substance is evidently not pure.

$$
\beta \text {-Pinosylvin monoglucoside }
$$

The above tetraacetyl- $\beta$-pinosylvin monobenzoate glucoside was treated with potassium ethoxide in ethanol and freed from potassium salts with perchloric acid in the ordinary way. The ethanol was evaporated and the residue washed with ether to remove ethyl benzoate. When dried over phosphorus pentoxide a slightly brown, amorphous powder was obtained. The material was soluble in acetic acid, slightly soluble in water and ethanol and insoluble in ether. On catalytic hydrogenation $0.93 \mathrm{mols}$ of hydrogen were consumed. All attempts to obtain this substance in a definitely crystalline state or in a state of higher purity failed. It was therefore acetylated, but yielded an amorphous product.

A small sample of the phenol glucoside preparation was methylated with diazomethane in methanol. Part of the methylated product was acetylated with acetic anhydride in pyridine. M. p. of the obtained glucoside $174-175^{\circ}$. Mixed m. p. with $\beta$ pinosylvin monomethyl ether glucoside $175-176^{\circ}$. M. p. of the obtained acetate 106$107^{\circ}$. Mixed m. p. with tetraacethyl- $\beta$-pinosylvin monomethyl ether glucoside $106-107^{\circ}$. The glucoside and its acetate from the above experiments proved, then, to be identical with the previously described $\beta$-pinosylvin monomethyl ether glucoside and its acetate.

$$
\text { B-Dihydropinosylvin monoglucoside }
$$

A small sample of crude pinosylvin monoglucoside was hydrogenated in ethanol with palladium on animal charcoal as catalyst. The solution was filtered and the ethanol evaporated. The residue was dissolved in methanol. When the methanol was allowed to evaporate at room temperature the glucoside crystallized in colourless needles.

The crystalline mass was washed with water and ether and recrystallized once more from methanol. M. p. of the thoroughly dried preparation $168-170^{\circ}$.

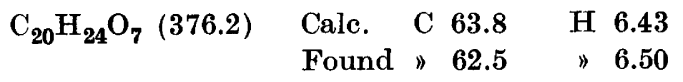

The substance evidently is not pure. The amount available, however, was too small to permit further purification. 


\section{SUMMARY}

The mono- and di- $\beta$-glucosides of pinosylvin and the $\beta$-glucoside of pinosylvin monomethyl ether have been prepared. The monoglucoside, however, could not be obtained in a state of purity.

I am indebted to Professor Holger Erdtman for suggesting this work and for helpful advice.

\section{REFERENCES}

1. Erdtman, H. Ann. 539 (1939) 116.

2. Kawamura, S. J. Pharm. Soc. Japan 58 (1938) 83 (Chem. Zentr. 1939, I, 130).

3. Erdtman, H. Svensk Papperstidn. 48 (1945) 217.

4. Bonner, W. A., and Koehler, W. L. J. Am. Chem. Soc. 70 (1948) 314.

Received November 8, 1948. 